\title{
(c) (1)(9)(2) \\ El autoconcepto y las habilidades sociales de los estudiantes de Psicopedagogía (Universidad Técnica de Ambato) durante la emergencia sanitaria
}

\author{
The self-concept and social skills of Psychopedagogy students (Technical \\ University of Ambato) during the health emergency
}

Bermeo Ochoa Joseline Andrea. ${ }^{1}$, Luis René Indacochea Mendoza. ${ }^{2}$, Gladys Alexandra Cadena Peralta. ${ }^{3} \&$ Melida Patricia Ochoa Guallpa. ${ }^{4}$

\begin{abstract}
.
Introduction. In the present study it is highlighted that people strengthen their personality through their self-concept and the acquisition of social skills, components that favor the assimilation of procedures, the development of skills, and reflection, articulating conceptual, procedural, and attitudinal knowledge to cope with a changing world. Objective. To determine the influence of Self-concept on the social skills of leveling students, first and second in the Psychopedagogy career of the Technical University of Ambato during the Health Emergency. Methodology. This research has a qualitativequantitative approach with a documentary and field bibliographic review through the application of the Test AF-5 Self-concept Form 5 and the Gismero Social Skills Scale. The RHO-Sperman statistic shows a slight inverse correlation between the Physical SelfConcept dimension and the Anger Expression area. Outcome. Chi-square approves the hypothesis: Self-concept IF affects the Social Skills of leveling students, first and second

1 Universidad Técnica de Ambato, Facultad de Ciencias Humanas y de la Educación. Carrera Psicopedagogía, Ambato, Ecuador, email: jbermeo7712@uta.edu.ec. Orcid: https://orcid.org/0000-00030619-2747

2 Universidad Técnica de Ambato. Facultad de Ciencias Humanas y de la Educación. Carrera Psicopedagogía, Ambato, Ecuador, email: luisrindacocheam@uta.edu.ec. Orcid: https://orcid.org/00000002-0215-5263

3 Universidad Técnica de Ambato. Facultad de Ciencias Humanas y de la Educación. Carrera Psicopedagogía, Ambato, Ecuador, email: gladyscadena98@gmail.com Orcid: https://orcid.org/00000002-0808-96232

${ }^{4}$ Unidad Educativa Julio C. Larrea. Ambato, Ecuador. email: melida.ochoa@educacion.gob.ec. Orcid: https://orcid.org/0000-0002-1089-6467
\end{abstract}


in the Psychopedagogy career during the Health Emergency. Based on the research, it is concluded that the students have a Medium Self-concept and Low Social Skills. Conclusion. The Department of Student Welfare is suggested to make use of it so that university students can raise their level of Self-concept and Social Skills, which will help them develop and function in different contexts such as: academic, social, personal and professional and so on. Meet your proposed goals in a specified time.

Keywords: Social Skills, Self-concept, Psychopedagogy, Correlation.

\section{Resumen.}

Introducción. En el presente estudio se resalta que las personas afianzan su personalidad mediante su autoconcepto y la adquisición de habilidades sociales, componentes que favorecen en la asimilación de procedimientos, el desarrollo de destrezas, y la reflexión, articulando el conocimiento conceptual, procedimental, y actitudinal para hacer frente a un mundo cambiante. Objetivo. Determinar la influencia del Autoconcepto en las habilidades sociales de los estudiantes de nivelación, primero y segundo de la carrera de Psicopedagogía de la Universidad Técnica de Ambato durante la Emergencia Sanitaria. Metodología. La presente investigación tiene enfoque cuali-cuantitativo con revisión bibliográfica documental y de campo mediante la aplicación del Test AF-5 Autoconcepto Forma 5 y Escala de Habilidades Sociales de Gismero. El estadígrafo RHO-Sperman arroja una correlación inversa leve entre la dimensión Autoconcepto Físico y el área Expresión de Enfado. Resultado. Chi-cuadrado aprueba la hipótesis: el Autoconcepto SI incide en las Habilidades Sociales de los estudiantes de nivelación, primero y segundo de la carrera de Psicopedagogía durante la Emergencia Sanitaria. En base a la investigación se concluye que los estudiantes tienen un Autoconcepto Medio y Habilidades Sociales Bajas. Conclusión. Se sugiere al Departamento de Bienestar Estudiantil hacer uso de la misma para que los estudiantes universitarios puedan elevar su nivel de Autoconcepto y Habilidades Sociales, mismos que le servirán para desarrollarse y desenvolverse en diferentes contextos como son: académico, social, personal y profesional y así cumplir sus metas propuestas en un tiempo determinado.

Palabras claves: habilidades Sociales, Autoconcepto, Psicopedagogía, Correlación.

\section{Introducción.}

El ser humano necesita poseer un bienestar biopsicosocial estable, mismo que le permitirá estar bien con sí mismo, el medio que le rodea y sobre todo tener un sano equilibrio emocional, todos estos relacionados con el Autoconcepto que se define como la capacidad que tiene un individuo para auto conocerse, logrando así describirse tanto en sus atributos físicos como comportamentales al igual que sus habilidades personales. 


\section{Autoconcepto}

Durante la adolescencia los jóvenes sufren varios cambios tanto físicos como sociales, con los que deben aprender a convivir para alcanzar la estabilidad emocional. Espejo et.al. (2018) afirma que "La etapa adolescente se destaca por ser una etapa fundamental en el afianzamiento de la personalidad, así como la adquisición de hábitos que se asentarán en la adultez" (pág. 1). Uno de estos factores a influir sin duda en el desarrollo de la personalidad y el funcionamiento integral del individuo es el Autoconcepto ya que es parte fundamental para un desarrollo óptimo de su bienestar social y personal (Patón, Ferreira, \& García, 2018)

\section{Definición de Autoconcepto}

El Autoconcepto antiguamente era definido como un constructo unitario que contemplaba el todo de una persona, pero hoy en día se define con una concepción multidimensional. El Autoconcepto tiene estrecha relación con el Autoestima que, por su parte, se relaciona con un componente afectivo evaluativo, mismo que nos permite amarnos y aceptarnos tal cual somos (Abilleira \& Rodicio, 2017). El Autoconcepto nos permite desarrollar la personalidad individual de las personas, de tal manera, el Autoconcepto tiene mucha trascendencia en otros aspectos de la vida, como pueden ser (Sailema, y otros, 2017)

\section{Formación del Autoconcepto}

Para el desarrollo del Autoconcepto es importante considerar las relaciones sociales que puede tener un individuo y a su vez asociarlo con su contexto ya sea familiar, educativo o profesional. El autoconcepto resulta de gran utilidad para entender el desarrollo durante la niñez media, pues interactúa con la personalidad y la conducta social. El niño se forma una imagen cada vez más estable de sí mismo, y su autoconcepto se vuelve más realista (Aranda \& Chávez, 2017). El autoconcepto cumple diferentes funciones: normaliza y guía los comportamientos, refuerza los estados afectivos, emociones y experiencias con las personas del entorno con el propósito de .alcanzar la satisfacción de las necesidades básicas del individuo (García, Mora, Valle, \& Ruiz, 2017)

\section{Tipos de Autoconcepto}

El autoconcepto se encuentra integrado por la siguiente tipología:

El Autoconcepto físico: tiene mucha relevancia con respecto a cómo se ve la persona físicamente y qué hace para sentirse bien consigo mismo. El Autoconcepto personal: hace referencia a la idea que tiene un individuo sobre sí mismo en cuanto a ser individual.El Autoconcepto social: se organiza dependiendo las competencias o habilidades que el individuo haya obtenido a lo largo de la vida en diferentes situaciones, tomando a consideración las habilidades sociales, la benevolencia, la agresividad, el asertividad, entre otras.El Autoconcepto académico: tiene que ver con la percepción de la capacidad o habilidad que tiene un individuo con respecto a las materias de un currículo establecido en el ámbito escolar (Esnaola, Goñi, \& Madariaga , 2008) 


\section{Autoconcepto y la Personalidad}

La personalidad es un aspecto central para la formación del autoconcepto ya que en conjunto estos predicen la satisfacción personal de la vida. El autoconcepto tiene que ver en gran parte según estudios con la cultura, ya que de la identidad cultural depende que las personas sean conscientes de su propia auto-descripción. De tal manera, el Autoconcepto es la opinión que tenemos de nosotros mismos, pero a su vez es un factor importante e imprescindible de la personalidad, ya que al trabajar de manera conjunta llegan a ser la pauta para interpretar y guiar sus propias experiencias vitales.

\section{Habilidades Sociales}

La situación actual del Ecuador y del mundo entero se ha visto afectada en varios ámbitos como son el laboral, académico, profesional y social, debido a la reciente Emergencia Sanitaria por Covid - 19, para lograr adaptarse a todas estas demandas es necesario un buen desarrollo de Habilidades Sociales que sin lugar a duda cada día se vuelve más difícil, ya que para su progreso se necesita de un aprendizaje individual asociado a un contexto y las relaciones sociales que un individuo pude tener (Cáceres, 2018)

\section{Definición de las Habilidades Sociales}

Las habilidades sociales hacen referencia a los comportamientos verbales y no verbales (expresión facial, postura, gestos, etc) que pueden suceder en diferentes circunstancias como la interacción social; a su vez permiten expresar libremente nuestras ideas, pensamiento, deseos o sentimientos, mismas que nos hacen sentir bien. También nos permiten aceptar y brindar críticas teniendo la capacidad de dejar pasar aquellas que no nos construyen, de tal manera no nos enfadamos sino más bien crecemos y continuamos nuestro camino o mejoramos.

\section{Adquisición de las Habilidades Sociales}

Se considera que un buen desarrollo social está basado en una buena adquisición de habilidades sociales, mismas que se van adquiriendo durante todo el ciclo de vida. Estas habilidades sociales datan desde el autoconocimiento, conocimiento social, el autocontrol, control y apoyo emocional, desarrollo moral hasta el descubrimiento de su preferencia sexual (Zumba, 2017)

La familia cumple un papel fundamental en el desarrollo de las Habilidades Sociales de un individuo ya que interfiere en la maduración del individuo de manera individual y grupal. Es por ello, que desde la perspectiva de la Psicología Social podemos considerar a la familia como el primer eje para que una persona logre tener relaciones sociales estables durante toda su vida y sobre todo pueda auto realizarse en el ámbito personal y profesional (Adarve, Ortega, Sánchez, Ruz, \& Sánchez , 2019) Los niños desde los cinco años tienen la predisposición y las ganas de colaborar, ya que según la formación de sus padres han logrado adquirir habilidades pro-sociales como el ayudar a adultos o amigos, 
compartir, hacer y pedir favores, jugar, ser amable, entre otras. Es por ello que según (Barona, 2021)

Tabla 1.

Elementos característicos de las Habilidades Sociales.

\begin{tabular}{ll}
\hline \multicolumn{1}{c}{ Elementos } & \multicolumn{1}{c}{ Características } \\
\hline Conductuales & Expresión facial. \\
& Postura corporal. \\
& Mirada y sonrisa. \\
& Orientación \\
& Comunicación verbal y no verbal. \\
& Preguntas y respuestas. \\
Cognitivos & Desarrollo de competencias. \\
& Estrategias de codificación. \\
Fisiológicos & Constructos personales. \\
& Flujo sanguíneo. \\
& Presión arterial. \\
& Frecuencia cardíaca. \\
& Tensión muscular. \\
\hline
\end{tabular}

Elaborado por: La investigadora

\section{Tipos de Habilidades Sociales}

Las habilidades sociales se dividen en seis grupos mismos que incluyen el desarrollo de habilidades y comportamientos que le permitan al individuo desarrollarse de manera óptima en un ambiente social (Rodríguez, Cacheiro, \& Gil, 2014)

Las habilidades sociales básicas: se basan principalmente en aspectos morales que le permiten al individuo crear vínculos interpersonales, de identificación y expresión tanto de sus emociones como intereses.

Las habilidades sociales avanzadas: estas por su parte permiten al individuo combinar las normas de comportamiento con la interacción social más sofisticada del mismo para su buen desenvolvimiento social.

Las habilidades relacionadas con los sentimientos son aquellas que permiten manejar las emociones y expresarlas socialmente de manera adecuada.

Las habilidades sociales alternativas: implican el uso de diferentes vías para abordar una situación en específico.

Las habilidades que permiten enfrentar el estrés: se refieren a la capacidad que tiene una persona para manejar de manera adecuada las tensiones y el estrés (Amaral, Maia, \& Bezerra, 2015)

Las habilidades de planificación: son las que permiten al individuo manejar su aburrimiento, identificar el porqué de un problema, buscar soluciones realistas, buscar información necesaria, priorizar la resolución de conflictos y sobre todo la habilidad de concentración. 


\section{Actividades para la adquisición y desarrollo de Habilidades Sociales}

Dentro de las Habilidades Sociales debemos considerar varias competencias y su desarrollo óptimo con diversas actividades que se relacionen desde muy temprana edad, es por ello que varios autores como (Moscoso, 2010) y organizaciones como "Mind the gap" mencionan diferentes competencias para desarrollar cada habilidad que se encuentra dentro de las Habilidades Sociales mismas que se detallarán a continuación:

Tabla 2.

Habilidades y Competencias Sociales.

\begin{tabular}{|c|c|}
\hline Habilidad & Competencias \\
\hline Comunicación & $\begin{array}{l}\text { Práctica de la escucha activa. } \\
\text { Práctica de la comunicación asertiva. } \\
\text { Tipos de comunicadores. } \\
\text { Expresión correcta. } \\
\text { Intervenciones oportunas para evitar conflictos. }\end{array}$ \\
\hline Empatía & $\begin{array}{l}\text { Ponerse en el lugar del otro. } \\
\text { Entender el punto de vista o perspectiva del otro. } \\
\text { Compartir sentimientos y emociones considerando el } \\
\text { contexto. } \\
\text { Ser conscientes de acciones inapropiadas que se pueden } \\
\text { cometer. }\end{array}$ \\
\hline Manejo de emociones & $\begin{array}{l}\text { Conoce sus emociones y los factores que influyen en sus } \\
\text { cambios. } \\
\text { Controlar sus cambios de humor. } \\
\text { Buscar tiempo fuera cuando se encuentra enfadado. } \\
\text { Controlar sentimientos negativos. } \\
\text { Identificar contextos que le pueden provocar } \\
\text { intranquilidad. }\end{array}$ \\
\hline Manejo de tensiones y estrés & $\begin{array}{l}\text { Realiza técnicas apropiadas para manejar su estrés. } \\
\text { Realizar actividades fuera de lo común. } \\
\text { Utilizar adecuadamente su tiempo libre. } \\
\text { Pedir ayuda en situaciones estresantes. }\end{array}$ \\
\hline Autoconocimiento o Autovaloración. & $\begin{array}{l}\text { Es conscientes de las consecuencias que puede acarrear } \\
\text { su comportamiento. } \\
\text { Evitar repetir los mismos errores. } \\
\text { Hace caso a las críticas constructivas que pueden hacerle. }\end{array}$ \\
\hline Toma de decisiones & $\begin{array}{l}\text { Piensa con cabeza fría antes de tomar una decisión. } \\
\text { Sabe los riesgos que puede traer una decisión. } \\
\text { Examina la información que tiene acerca de la decisión } \\
\text { antes de tomarla. } \\
\text { Escucha opiniones de los demás. }\end{array}$ \\
\hline Resolución de Conflictos & $\begin{array}{l}\text { Identifica el problema. } \\
\text { Se da un tiempo para meditar el problema. } \\
\text { Busca soluciones a los problemas. } \\
\text { Practica comunicación asertiva y escucha activa. } \\
\text { Es consciente del grado de afectación que puede dejar un } \\
\text { problema. }\end{array}$ \\
\hline Trabajo colaborativo o en equipo. & $\begin{array}{l}\text { Sabe manejar a un grupo. } \\
\text { Es un líder más un jefe. } \\
\text { Colabora en un grupo. }\end{array}$ \\
\hline
\end{tabular}


Tabla 2.

Habilidades y Competencias Sociales. (continuación)

\begin{tabular}{ll}
\hline \multicolumn{1}{c}{ Habilidad } & \multicolumn{1}{c}{ Competencias } \\
\hline Trabajo colaborativo o en equipo. & Reconoce cuando los compañeros tienen éxito. \\
Derechos Humanos e Interculturalidad & Reconoce y respeta los derechos propios y de los demás. \\
& Respeta culturas. \\
& Evita la discriminación. \\
& Tiene un enfoque de inclusión. \\
\hline
\end{tabular}

Elaborado por: La investigadora

\section{El Autoconcepto en las Habilidades Sociales}

El autoconcepto es un constructo fundamental para el desarrollo personal de una persona, siendo así que, si una persona tiene un autoconcepto definido será capaz de tomar mejores decisiones con respecto a su proyecto de vida, se desenvolverá de manera acertada en un contexto social y manejará correctamente sus relaciones sociales (Espejo, Zurita, Chacón, Castro, \& Martínez, 2018)

Las Habilidades Sociales por su parte, son conductas que se relacionan entre sí y nos permiten relacionarnos con otras personas en diferentes situaciones y contextos, como bien sabemos, somos seres sociales desde el momento en que nacemos y conocemos por primera vez a nuestros padres, es por ello que la práctica adecuada de las habilidades sociales nos permitirá crear lazos de empatía, solidaridad, trabajo colaborativo, resolución de problemas, manejo de emociones, entre otras de manera congruente (Cáceres, 2018)

De igual manera nos menciona que en medida en que el autoconcepto de un individuo se fortalece de manera positiva, sus habilidades sociales lo harán de la misma manera, dejando a evidencia la estrecha relación entre las variables de estudio de este trabajo investigativo. Finalmente, se pone a consideración a los centros educativos, el trabajar en actividades y acciones formativas que desarrollen y fortalezcan tanto el Autoconcepto como las Habilidades Sociales para potencializar las destrezas y habilidades, a su vez evitar comportamientos disfuncionales o patologías personales.

\section{Metodologia.}

\section{Enfoque}

El enfoque de investigación que se ejecutó con las variables de autoconcepto y habilidades sociales tuvo carácter cuali-cuantitativo o mixto.Cualitativo, a través del mismo se pudo recopilar información, realizar un análisis y narrar aspectos teóricos tanto del autoconcepto como las habilidades sociales desde la perspectiva de varios autores sin implicar mediciones numéricas.

Cuantitativo, debido a que se evaluó el nivel de autoconcepto mediante el Test AF-5 autoconcepto forma $5 \mathrm{y}$ el nivel de habilidades sociales mediante la escala de habilidades sociales de Gismero, mismos que arrojaron datos estadísticos que permitieron la recolección de información numérica y estadística. 


\section{Alcances de la investigación}

De acuerdo a los alcances de investigación que menciona Hernández (2014), se utilizaron los siguientes:

Descriptiva, debido a que se detalló la incidencia del Autoconcepto en las Habilidades Sociales de los estudiantes de la carrera de Psicopedagogía de la Universidad Técnica de Ambato, a su vez permitió recolectar datos para posteriormente clasificar, ordenar, analizar y describir; en tal virtud se determinó características en diferentes situaciones y contextos del ámbito psicopedagógico.

Explicativa, ya que se manifestó el porqué del fenómeno social y las condiciones en las que se da la incidencia del Autoconcepto en las Habilidades Sociales de los estudiantes de la carrera de Psicopedagogía de la Universidad Técnica de Ambato.

Correlacional, ya que se pudo conocer el grado de asociación entre las variables, logrando identificar si la incidencia del Autoconcepto tiene que ver con el desarrollo óptimo de las Habilidades Sociales de los estudiantes de la carrera de Psicopedagogía de la Universidad Técnica de Ambato.

\section{Modalidad de Investigación}

Según Baena (2017) la modalidad de la investigación se divide en dos grandes grupos la documental y de campo, mismos que se utilizaron en la investigación y se detallan a continuación:

Bibliográfico documental, debido a que esta investigación fue de carácter virtual y se realizó a partir de la revisión de diferentes fuentes bibliográficas o documentales, a su vez predominó el análisis, la interpretación, las opiniones y conclusiones acerca del Autoconcepto y las Habilidades Sociales de los estudiantes de la carrera de Psicopedagogía de la Universidad Técnica de Ambato.

De campo, ya que se obtuvo datos veraces y directos con los sujetos a investigar al igual que su realidad en el contexto determinado, plasmando la información obtenida a través del test y escala sin ninguna alteración. Sin embargo, debido a la reciente emergencia Sanitaria por Covid-19, se adaptó a medios digitales online como es Google Forms para recabar dicha información.

\section{Población y muestra}

La población del presente trabajo investigativo estuvo constituida por los estudiantes de Nivelación, Primero y Segundo de la carrera de Psicopedagogía de la Facultad de Ciencias Humanas y de Educación de la Universidad Técnica de Ambato. Esta investigación tuvo una muestra de 110 estudiantes. 
Tabla 3.

Población

\begin{tabular}{llll}
\hline \multicolumn{1}{c}{ Unidades de observación } & Frecuencia & Porcentaje \\
\hline Estudiantes & 110 & $100 \%$ \\
Total & $\mathbf{1 1 0}$ & $\mathbf{1 0 0 \%}$
\end{tabular}

Fuente: Facultad de Ciencias Humanas y de la Educación. Universidad Técnica de Ambato.

Elaborado por: La investigadora

\section{Técnica}

La técnica utilizada para el presente trabajo de investigación tuvo un enfoque psicométrico basado en la aplicación de Test para cada una de las variables, mismos que permitieron evaluar el nivel de Autoconcepto y Habilidades Sociales en los estudiantes de Nivelación, Primero y Segundo de la carrera de Psicopedagogía, para posterior analizar, organizar y clasificar la información obtenida y poder brindar conclusiones y recomendaciones que fueron elaboradas tomando como base los objetivos propuestos en el estudio.

\section{Instrumento}

En el presente trabajo de investigación se utilizó dos instrumentos diferentes que permitieron obtener la información de las variables, mismos que fueron adaptados a plataformas online para su posterior aplicación.

\section{AF-5. Autoconcepto Forma 5}

Autor: (García \& Musitu, 2014)

Aplicación: Individual o colectiva.

Población: Niños/as y adultos. De los 10 años en adelante.

Duración: Aproximadamente 15 minutos.

Finalidad: Para evaluar el Autoconcepto se utilizó el Test AF-5 Autoconcepto Forma 5, mismo que evaluó el Autoconcepto mediante 30 elementos.

Se dividen en el área social, académico/profesional, emocional, físico y familiar, brindando de esta manera aspectos clave para el buen desarrollo biopsicosocial de los estudiantes.Esencialmente, una de las funciones psicológicas de mayor trascendencia atribuida a la familia es la formación del autoconcepto o identidad de sus integrantes (García \& Musitu, 2014)

\section{EHS. Escala de Habilidades Sociales}

Autora: (Gismero, 2010)

Aplicación: Individual o colectiva. 
Población: Adolescentes y Adultos.

Duración: Aproximadamente 16 minutos.

Finalidad: En cuanto refiere a las Habilidades Sociales se aplicó la Escala de Habilidades Sociales de Gismero.

Está compuesta por 33 ítems divididos en: 28 ítems para déficit en habilidades sociales y los 5 restantes tienen un enfoque positivo.

\section{Resultados}

Después de la respectiva aplicación de los instrumentos a una población de 110 estudiantes de nivelación, primero y segundo de la carrera de Psicopedagogía de la Facultad de Ciencias Humanas y de la Educación de la Universidad Técnica de Ambato, se procede a analizar e interpretar los datos obtenidos, considerando que el Test AF-5 Autoconcepto Forma 5 consta de 30 ítems mismos que están divididos en 5 dimensiones respectivamente y la Escala de Habilidades Sociales de Gismero conformada por 33 ítems repartidos en 6 subáreas.

\section{Resultado Test AF-5 Autoconcepto Forma 5.}

Figura 1.

Porcentajes por dimensiones. AF-5. Autoconcepto Forma 5

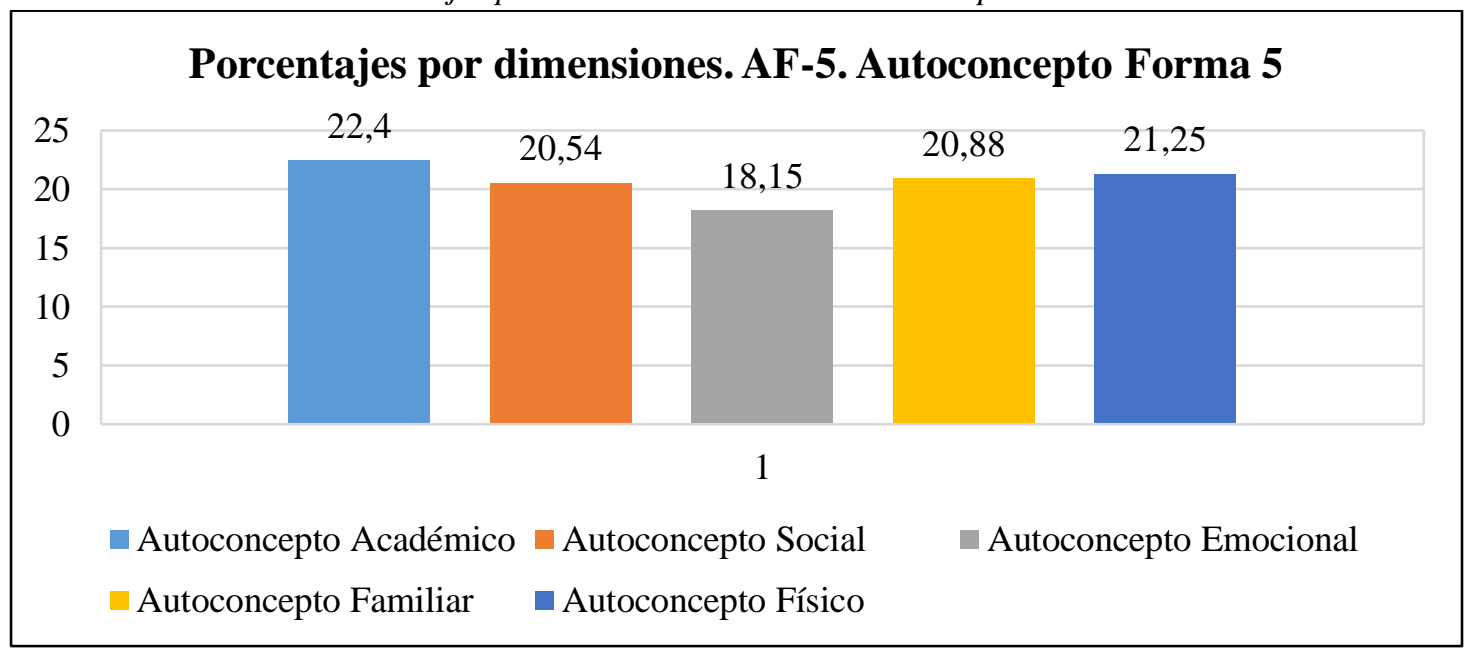

Gráfico 1. Dimensiones Autoconcepto según Test AF-5

Elaborado por: La investigadora

Dentro de las dimensiones generales que se encuentran en el Test AF-5 Autoconcepto Forma 5, después de la aplicación y asociación de preguntas, se obtiene los siguientes resultados: 22,4 \% para Autoconcepto Académico, 20,55\% para Autoconcepto Social; $18,15 \%$ para Autoconcepto Emocional; 20,88\% para Autoconcepto Familiar y por último 21, 25\% para Autoconcepto Físico.

De acuerdo a los porcentajes obtenidos en cada una de las dimensiones del Test se obtuvieron los siguientes resultados: en primera instancia presentan un porcentaje alto en la dimensión Autoconcepto Académico mismo que da a conocer su propia percepción 
con respecto a los trabajos escolares, ser buen estudiante, trabajador, inteligente, líder y participativo, motivo por el cual sus docentes lo estiman y hay una buena relación entre pares.

Seguido se encuentra el Autoconcepto Físico, donde los estudiantes dan a conocer su cuidado personal, práctica deportiva y su percepción física con respecto a la elegancia y ser atractivo, esto les genera sentimientos de autoestima personal; por su parte dentro de la tercer área con relevancia tenemos el autoconcepto familiar, que va enmarcado a los sentimientos positivos que generan el estar en casa, la confianza en familia, el cariño y la forma de solventar problemas con el apoyo familiar.

Por otra parte tenemos autoconcepto social, donde lo estudiantes dieron a conocer su forma de adaptarse al medio, la manera de hacer amigos y relacionarse con los mismo; y por último el autoconcepto emocional, este tiene un promedio bajo y tiene que ver con las situaciones adversas que pueden provocar cambios emocionales en los estudiantes.

\section{Resultados de Escala de Habilidades Sociales de Gismero}

Escala de Habilidades Sociales

Figura 2.

Escala de habilidades sociales

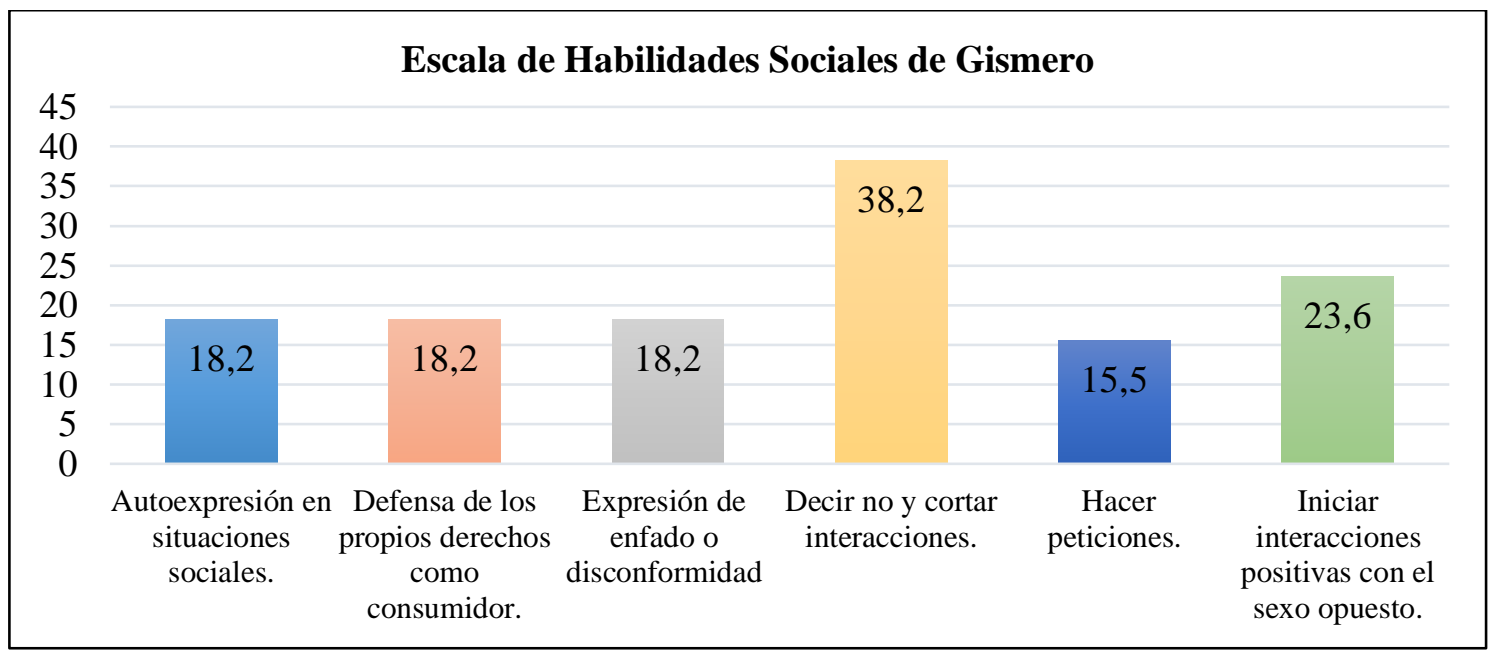

Elaborado por: La investigadora

Dentro de las áreas generales que se encuentran en la Escala de Habilidades Sociales de después de la aplicación y asociación de preguntas, se obtiene los siguientes resultados: $18,2 \%$ para área 1. Autoexpresión en situaciones sociales, 18,2\% para área 2. Defensa de los propios derechos como consumidor; 18,2\% para área 3. Expresión de enfado o disconformidad; 38,2\% para área 4. Decir no y cortar interacciones; $15,5 \%$ para área 5. Hacer peticiones y por último $23,6 \%$ para área 6 . Iniciar interacciones positivas con el sexo opuesto.

De acuerdo a los porcentajes se obtuvieron los siguientes resultados: 1. Autoexpresión en situaciones sociales, misma que se relaciona con la habilidad de hacer preguntas en diferentes lugares como reuniones o la escuela, hacer entrevistas personales o expresar 
opiniones sin sentirse turbado, el área 2. Defensa de los propios derechos como consumidor, en esta se inmiscuye el hecho de devolver algo que me vendieron mal, solicitar silencio en el cine, pedir descuentos o solicitar que se respete la fila del supermercado, en el área 3. Expresión de enfado o disconformidad, donde los estudiantes se expresan de manera libre ante situaciones de desagrado o que a su vez le causan molestia; y finalmente el área con menor puntaje es área 5.

\section{Correlación RHO de Spearman.}

Tabla 4.

Correlación entre las dimensiones de autoconcepto y las áreas de habilidades sociales.

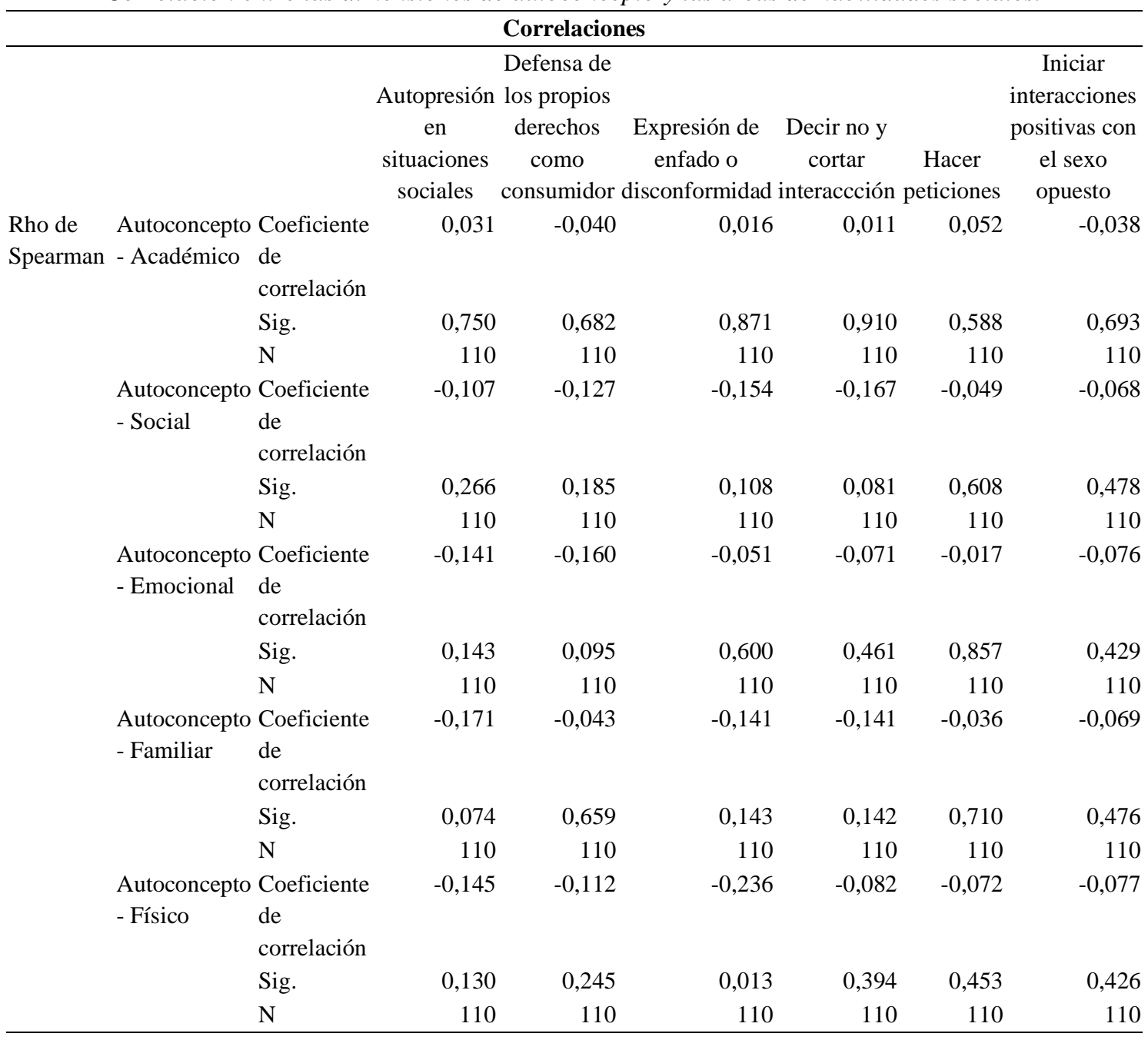

Fuente: Correlación: autoconcepto y habilidades sociales

Elaborado por: La investigadora

Dentro de la correlación entre las dimensiones del autoconcepto y las áreas de habilidades sociales se encuentran los siguientes resultados, tomando a consideración que una significación mayor a 0,05 anula la correlación (Franco, 2006)

Autoconcepto Académico con Autoexpresión en situaciones sociales 0,750; Autoconcepto Académico con Defensa de los propios derechos como consumidor 0,682; Autoconcepto Académico con Expresión de enfado o disconformidad 0,871; 
Autoconcepto Académico con Decir no y cortar interacciones 0,910; Autoconcepto Académico con Hacer peticiones 0,588 y Autoconcepto Académico con Iniciar interacciones positivas con el sexo opuesto 0,693 .

Autoconcepto Social con Autoexpresión en situaciones sociales 0,266; Autoconcepto Social con Defensa de los propios derechos como consumidor 0,185; Autoconcepto Social Expresión de enfado o disconformidad 0,108; Autoconcepto Social con Decir no y cortar interacciones 0,081; Autoconcepto Social con Hacer peticiones 0,608 y Autoconcepto Social con Iniciar interacciones positivas con el sexo opuesto 0,478 (Delgado, M., Pequeña, Rodríguez, \& Álvarez, 2016).

Autoconcepto Emocional con Autoexpresión en situaciones sociales 0,143; Autoconcepto Emocional con defensa de los propios derechos como consumidor 0,095; autoconcepto emocional expresión de enfado o disconformidad 0,600; autoconcepto emocional con decir no y cortar interacciones 0,461 ; autoconcepto emocional con hacer peticiones 0,857 y autoconcepto emocional con iniciar interacciones positivas con el sexo opuesto 0,429 (Caldera, Reynoso, Angulo, Cadena, \& Ortíz, 2018).

Autoconcepto familiar con autoexpresión en situaciones sociales 0,074; autoconcepto familiar con defensa de los propios derechos como consumidor 0,659 ; autoconcepto familiar con expresión de enfado o disconformidad 0,143; autoconcepto familiar con decir no y cortar interacciones 0,142 ; autoconcepto familiar con hacer peticiones $0,710 \mathrm{y}$ autoconcepto familiar con iniciar interacciones positivas con el sexo opuesto 0,476 .

Autoconcepto físico con autoexpresión en situaciones sociales 0,130; autoconcepto físico con defensa de los propios derechos como consumidor 0,245; autoconcepto físico con expresión de enfado o disconformidad 0,013; autoconcepto físico con decir no y cortar interacciones 0,394 ; autoconcepto físico con hacer peticiones 0,453 y autoconcepto físico con iniciar interacciones positivas con el sexo opuesto 0,426. (Ruiz \& Jaramillo, 2010)

De acuerdo a las respuestas obtenidas en la correlación de las dimensiones de Autoconcepto y áreas de Habilidades Sociales según el estadígrafo HRO de Sperman se obtiene una correlación inversa leve entre autoconcepto físico y expresión de enfado o disconformidad lo que quiere decir que, si el autoconcepto físico baja, la expresión de enfado o disconformidad sube y viceversa, esta correlación tiene un alto nivel de lógica ya que, si el estudiante se siente feo y nada elegante va a mostrar molestia que se somatizará en enfado e incluso sentimientos de tristeza. (Tacca, Cuarez, \& Quispe, 2020)

\section{Verificación de la hipótesis}

Comprobación de la hipótesis con chi-cuadrado (X2).

\section{Planteamiento de la Hipótesis}

H0.: El Autoconcepto NO incide en las Habilidades Sociales de los estudiantes de nivelación, primero y segundo de la carrera de Psicopedagogía de la Universidad Técnica de Ambato, durante la Emergencia Sanitaria. 
H1.: El Autoconcepto SI incide en las Habilidades Sociales de los estudiantes de nivelación, primero y segundo de la carrera de Psicopedagogía de la Universidad Técnica de Ambato, durante la Emergencia Sanitaria.

\section{Selección del nivel de significación}

En la realización de la investigación el nivel de confianza que se tendrá es del 95\%, por lo que el nivel de riesgo será de 5\%. Para la verificación de la hipótesis se utilizará el nivel de $\alpha=0.05$. Se ha tomado como referencia para la investigación de campo la población total de 110 estudiantes de nivelación, primero y segundo de la carrera de Psicopedagogía de la Universidad Técnica de Ambato.

Tabla 5.

Población y porcentaje.

\begin{tabular}{ll}
\hline Población & 110 \\
Porcentaje & $100 \%$
\end{tabular}

Elaborado por: La investigadora

Es necesario mencionar que para la verificación de la hipótesis se expresará un cuadro de contingencia de 4 filas por 4 columnas con las que se determinarán las frecuencias esperadas.

Tabla 6.

Especificación de lo estadístico.

\begin{tabular}{llllll}
\hline \multicolumn{1}{r}{ ITEMS } & \multicolumn{2}{c}{ ESCALAS } & & \multicolumn{2}{c}{ SUBTOTAL } \\
\cline { 3 - 5 } & $\mathbf{S}$ & $\mathbf{C S}$ & $\mathbf{C N}$ & $\mathbf{N}$ & \\
\cline { 1 - 3 } & 11 & 73 & 17 & 4 & 110 \\
28 & 10 & 71 & 20 & 9 & 110 \\
18 & 13 & 23 & 25 & 48 & 110 \\
27 & 24 & 33 & 28 & 25 & 110 \\
TOTAL & $\mathbf{5 8}$ & $\mathbf{2 0 0}$ & $\mathbf{9 0}$ & $\mathbf{8 6}$ & $\mathbf{4 3 4}$ \\
\hline
\end{tabular}

Elaborado por: La investigadora

Tabla 7.

Frecuencias Esperadas.

\begin{tabular}{lc}
\hline & Frecuencias Esperadas \\
\hline NO*Subtotal $) /$ Total & \\
$(58 * 110) / 434=$ & $\mathbf{1 4 , 7}$ \\
$(200 * 110) / 434=$ & $\mathbf{5 0 , 7}$ \\
$(90 * 110) / 434=$ & $\mathbf{2 2 , 8}$ \\
$(86 * 110) / 434=$ & $\mathbf{2 1 , 8}$ \\
TOTAL $=$ & $\mathbf{1 1 0}$ \\
\hline
\end{tabular}

Elaborado por: La investigadora

Especificación de las regiones de aceptación y rechazo. Se procede a determinar los grados de libertad considerando que el cuadro consta de 4 filas y 4 columnas. 
Tabla 8.

Grados de libertad.

GRADO DE LIBERTAD

\section{FILAS}

gl=

$\mathbf{g l}=$

$\mathbf{g l}=$

$\mathbf{g l}=$
(4-1)

3
COLUMNAS

(4-1)

3

$3 * 3$

9

Elaborado por: La investigadora

Por lo tanto, con 9 grados de libertad y a nivel 0.05 de significación según la tabla: X2T= 16,92. Por tanto, si $\mathrm{X} 2 \mathrm{C} \leq \mathrm{X} 2 \mathrm{~T}$ se aceptará la $\mathrm{H} 0$, caso contario se la rechazará y se aceptará la hipótesis alternativa.

Recolección de datos y cálculo de lo estadístico.

Para esto se utilizará la siguiente fórmula: $\mathrm{X} 2=\sum(\mathrm{O}-\mathrm{E}) 2$

Tabla 9.

Recolección de datos y cálculo de lo estadístico.

\begin{tabular}{llllll}
\hline ITEMS & $\mathbf{O}$ & $\mathbf{E}$ & $\mathbf{( O - E )}$ & $\mathbf{( O - E ) 2}$ & $(\mathbf{O - E}) \mathbf{2} / \mathbf{E}$ \\
\hline 8 & 11 & 14,7 & $-3,7$ & 13,7 & 0,9 \\
8 & 73 & 50,7 & 22,3 & 497,3 & 9,8 \\
8 & 17 & 22,8 & $-5,8$ & 33,64 & 1,5 \\
8 & 4 & 21,8 & $-17,8$ & 316,8 & 14,5 \\
28 & 10 & 14,7 & $-4,7$ & 22,09 & 1,5 \\
28 & 71 & 50,7 & 20,3 & 412,09 & 8,1 \\
28 & 20 & 22,8 & $-2,8$ & 7,84 & 0,3 \\
28 & 9 & 21,8 & $-12,8$ & 163,84 & 7,5 \\
18 & 13 & 14,7 & $-1,7$ & 2,89 & 0,19 \\
18 & 23 & 50,7 & $-27,7$ & 767,29 & 15,13 \\
18 & 25 & 22,8 & 2,2 & 4,84 & 0,21 \\
18 & 48 & 21,8 & 26,2 & 686,44 & 31,48 \\
27 & 24 & 14,7 & 9,3 & 86,49 & 5,88 \\
27 & 33 & 50,7 & $-17,7$ & 313,29 & 6,18 \\
27 & 28 & 22,8 & 5,2 & 27,04 & 1,19 \\
27 & 25 & 21,8 & 3,2 & 10,24 & 0,47 \\
& $\mathbf{4 3 4}$ & $\mathbf{4 4 0}$ & & $\mathbf{X}^{\mathbf{2}} \mathbf{C}=$ & \\
\hline
\end{tabular}

Elaborado por: La investigadora

Como se puede observar X2 calculado $=104,83$ es mayor que X2tabular $=16,92$ por tanto se acepta la hipótesis alterna que establece: H1: El Autoconcepto si incide en las habilidades sociales de los estudiantes de nivelación, primero y segundo de la carrera de psicopedagogía de la universidad técnica de Ambato, durante la emergencia sanitaria. 
Figura 3.

Correlación: autoconcepto y habilidades sociales

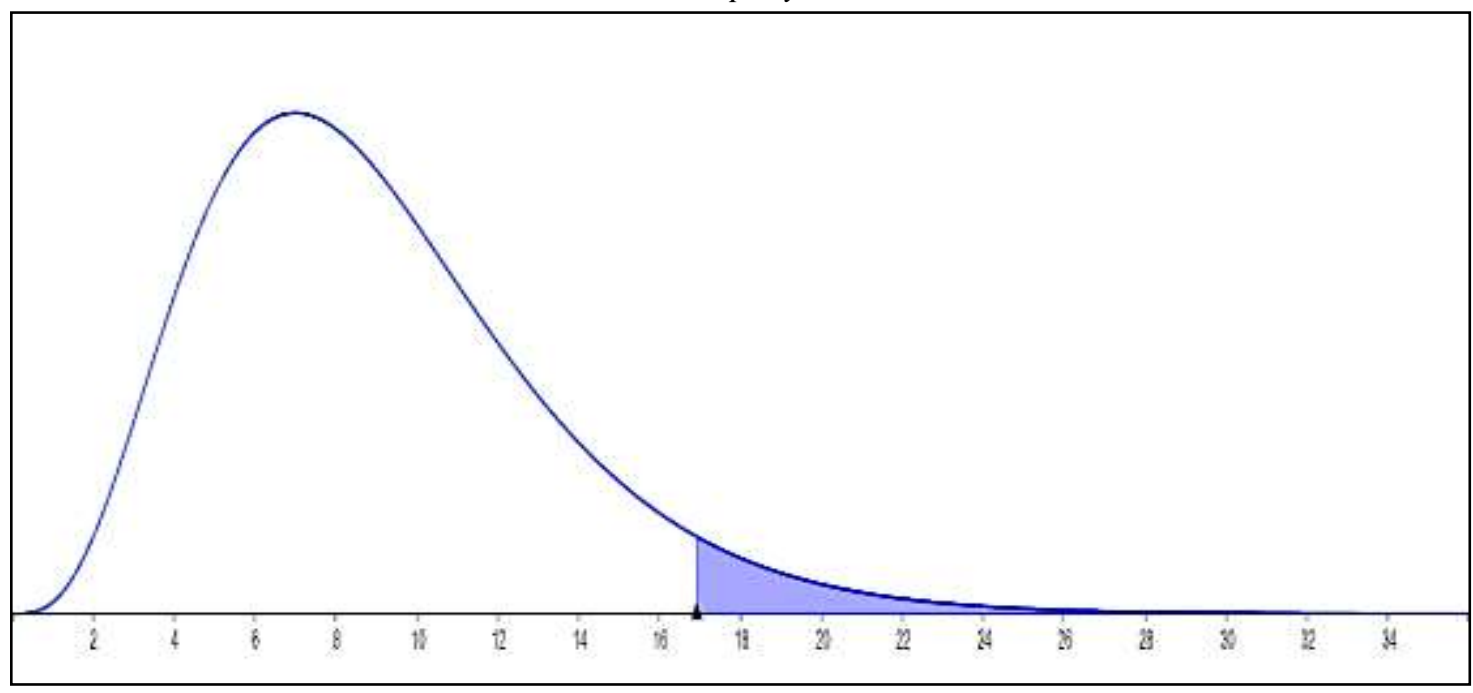

Elaborado por: La investigadora

\section{Discusión}

Después de la respectiva aplicación de los instrumentos a una población de 110 estudiantes de nivelación, primero y segundo de la carrera de Psicopedagogía de la Facultad de Ciencias Humanas y de la Educación de la Universidad Técnica de Ambato, se procede a analizar e interpretar los datos obtenidos, considerando que el Test AF-5 Autoconcepto Forma 5 consta de 30 ítems mismos que están divididos en 5 dimensiones respectivamente y la Escala de Habilidades Sociales de Gismero conformada por 33 ítems repartidos en 6 subáreas.

Para el análisis del coeficiente de correlación entre las dimensiones y áreas de los test psicométricos aplicados, se empleó RHO de Spearman, y así comprobar si existe asociación alguna. Este coeficiente es una medida de asociación lineal que utiliza los rangos y posteriormente los compara.

Por otro lado, se aplicó la prueba de Chi-Cuadrado para medir el coeficiente de correlación entre los resultados generales de las dos variables y así comprobar la hipótesis; esta es una prueba de estadística descriptiva que analiza datos nominales o cualitativos.

\section{Análisis}

Dentro de las dimensiones generales que se encuentran en el Test AF-5 Autoconcepto Forma 5, después de la aplicación y asociación de preguntas, se obtiene los siguientes resultados: 22,4 \% para Autoconcepto Académico, 20,55\% para Autoconcepto Social; 18,15\% para Autoconcepto Emocional; 20,88\% para Autoconcepto Familiar y por último 21, 25\% para Autoconcepto Físico.

Dentro del análisis general de las puntuaciones y resultados obtenidos con respecto a la variable Autoconcepto se obtiene lo siguiente: $0,9 \%$ de la población de estudiantes 
abordados señala un Autoconcepto Bajo; 72,7\% indica un Autoconcepto Medio y 26,4\% muestra un Autoconcepto Alto.

Dentro de las áreas generales que se encuentran en la Escala de Habilidades Sociales de Gismero, después de la aplicación y asociación de preguntas, se obtiene los siguientes resultados: 18,2\% para área 1. Autoexpresión en situaciones sociales, 18,2\% para área 2. Defensa de los propios derechos como consumidor; 18,2\% para área 3. Expresión de enfado o disconformidad; 38,2\% para área 4. Decir no y cortar interacciones; 15,5\% para área 5. Hacer peticiones y por último $23,6 \%$ para área 6 . Iniciar interacciones positivas con el sexo opuesto.

Dentro del análisis general de las puntuaciones y resultados obtenidos con respecto a la variable habilidades sociales se obtiene lo siguiente: $45,5 \%$ de la población de estudiantes abordados puntúa bajo en habilidades sociales; $36,4 \%$ puntúa medio en habilidades sociales y $18,2 \%$ puntúa alto en habilidades sociales.

\section{Interpretación}

De acuerdo a los porcentajes obtenidos en cada una de las dimensiones del Test se obtuvieron los siguientes resultados: en primera instancia presentan un porcentaje alto en la dimensión Autoconcepto Académico mismo que da a conocer su propia percepción con respecto a los trabajos escolares, ser buen estudiante, trabajador, inteligente, líder y participativo, motivo por el cual sus docentes lo estiman y hay una buena relación entre pares; seguido se encuentra el Autoconcepto Físico, donde los estudiantes dan a conocer su cuidado personal, práctica deportiva y su percepción física con respecto a la elegancia y ser atractivo, esto les genera sentimientos de autoestima personal

De acuerdo a la puntuación global obtenida de la aplicación del Test AF-5 Autoconcepto Forma 5, se obtuvo un nivel Medio de Autoconcepto como puntuación global, dicho resultado según investigaciones se encuentra bajo la norma, lo que permite a los estudiantes manejar todas las dimensiones del Autoconcepto de manera adecuada y en base a sus necesidades, logrando buena relación con el ámbito educativo, sus pares y docentes, hacer amigos con facilidad y relacionarse con los mismos, controlar sus emociones en situaciones difíciles, acogida familiar y apoyo con los mismos y por último su físico y autoestima que este provoca en los estudiantes (García, Mora, Valle, \& Ruiz, 2017).

De acuerdo a los porcentajes obtenidos en cada una de las áreas de la Escala de Habilidades Sociales de Gismero se obtuvieron los siguientes resultados: en primera instancia presentan un porcentaje alto en el área la dimensión Autoconcepto De acuerdo a las puntuaciones obtenidas dentro del área 4.

Decir no y cortar interacciones, que tiene relación con actividades del diario vivir que suelen ser incómodas para las personas, como un vendedor ambulante, una amiga que habla mucho o una persona con la que no me siento bien al compartir tiempo; seguido se encuentra el área 6. 
Iniciar interacciones positivas con el sexo opuesto, donde los estudiantes expresaron su habilidad para decirle a alguien que es atractivo, hacerle cumplidos, brindarle halagos o pedirle simplemente una cita; a continuación, se encuentran 3 áreas que obtuvieron el mismo resultado en el nivel Alto las cuales son área 1.

Autoexpresión en situaciones sociales, misma que se relaciona con la habilidad de hacer preguntas en diferentes lugares como reuniones o la escuela, hacer entrevistas personales o expresar opiniones sin sentirse turbado, el área 2. Defensa de los propios derechos como consumidor, en esta se inmiscuye el hecho de devolver algo que me vendieron mal, solicitar silencio en el cine, pedir descuentos o solicitar que se respete la fila del supermercado, en el área 3.

Expresión de enfado o disconformidad, donde los estudiantes se expresan de manera libre ante situaciones de desagrado o que a su vez le causan molestia; y finalmente el área con menor puntaje es área 5. Hacer peticiones que le permite al individuo pedir algo que dejo prestado, pedir que la orden de comida sea devuelta en caso de estar mal, pedir el cambio correctamente o pedirle un favor a un amigo. Sin embargo, estas puntuaciones no son representativas para afirmar un nivel alto en Habilidades Sociales de los estudiantes universitarios.

\section{Conclusiones}

- De acuerdo a la investigación realizada se concluye que existe un porcentaje importante de estudiantes que presentan rasgos de Autovaloración dentro de la norma, sin embargo, cabe resaltar que se encontró estudiantes con un nivel de Autoconcepto bajo, lo cual se evidencia en el aula de clase, en las pocas participaciones cuando se abordan temas desconocidos somatizando con rasgos de nerviosismo y en algunos casos particularmente en las féminas con rasgos de ansiedad y episodios eritrosicos e hiperhidrosicos.

- Por otro lado, dentro de la correlación de variables se encuentra que el Autoconcepto incide en las Habilidades Sociales de los estudiantes universitarios, siendo de tal forma que, en base a la percepción propia del individuo, este puede fortalecer su manera de manejar la vida social en diferentes contextos y en base a sus necesidades, logrando alcanzar sus objetivos propuestos de manera positiva. Además de la correlación inversa leve entre dimensión Autoconcepto Físico y área de enfado disconformidad, siendo así que a mayor percepción física menor disconformidad y viceversa.

- Finalmente, el presente proyecto de investigación permitió identificar las características relevantes del Autoconcepto y las Habilidades Sociales, así como sus dimensiones y áreas a trabajar para lograr un desenvolvimiento óptimo y favorable de los jóvenes universitarios a lo largo de su vida, permitiendo que su percepción académica, social, emocional, familiar y física tengan incidencia en el desarrollo de habilidades para cortar interacciones, hacer peticiones, desarrollo de la autoexpresión, expresión de enfado, entre otros, que a su vez facilitará la convivencia grupal e individual. 


\section{Bibliografía}

Abilleira, M., \& Rodicio, M. (2017). Análisis del autoconcepto en las víctimas de violencia de género entre adolescentes. Suma Psicológica,, 24(2), 107 - 114.

Adarve, M., Ortega, F., Sánchez, V., Ruz, R., \& Sánchez, A. (2019). Influencia de la práctica de actividad física en el autoconcepto de adolescente. Retos: nuevas tendencias en educación física, deporte y recreación., (36), 342 - 347.

Amaral, P., Maia, F., \& Bezerra, C. (2015). Las habilidades y el comportamiento infractor en la adolescencia. Subjetividad y Procesos Cognitivos.

Aranda , C., \& Chávez, L. (2017). Autoconcepto y habilidades sociales en niños como hijos únicos y niños con hermanos. PsicoEducativa: reflexiones y propuestas, 3(5), 38 - 44.

Baena, M. (2017). Metodología de la investigación (ea. ed.). Retrieved from http://ebookcentral.proquest.com Created from bibliotecacijsp on 2018-07-30 15:51:39.

Barona, E. (2021). La inteligencia emocional en el desarrollo de las habilidades sociales en estudiantes de primero y segundo semestre de la carrera de Psicopedagogía de la Universidad Técnica de Ambato. Ambato: Universidad Técnica dee Ambato.

Cáceres, M. (2018). El Autoconcepto en las habilidades sociales en estudiantes de educación secundaria de una institución educativa. Intervención del niño adolecente. Perú.

Caldera, J., Reynoso, O., Angulo, M., Cadena, A., \& Ortíz, D. (2018). Habilidades sociales y autoconcepto en estudiantes universitarios de la región Altos Sur de Jalisco. Escritos de Psicología - Psychological Writings, 11(3), 144-153.

Delgado, A., M., A., Pequeña, J., Rodríguez, R., \& Álvarez, D. (2016). Las habilidades sociales y el uso de redes sociales virtuales en estudiantes universitarios de Lima Metropolitana. Universidad de Lima, 55-75.

Esnaola, I., Goñi, A., \& Madariaga , J. (2008). El Autoconcepto: perspectivas de Investigación. Revista de Psicodidáctica, vol 13, núm 1., 69 - 96.

Espejo, T., Zurita, F., Chacón, R., Castro, M., \& Martínez, A. (2018). Actividad física y autoconcepto: dos factores de estudio en adolescentes de Zona rural.

Franco, J. (2006). Relación entre las variables autoconcepto y creatividad en una muestra de alumnos de educación infantil. 8(1), 16.

García, A., Mora, P., Valle, C., \& Ruiz, J. (2017). Autoconcepto en adultos: estudio de género y de tenencia de hermanos. Revista "Alternativas en Psicología”, 21(38), 33. 
García, F., \& Musitu, G. (2014). Autoconcepto Forma 5 (AF-5). TEA. Ediciones. Obtenido de http://www.web.teaediciones.com/ejemplos/af5_manual_2014_extracto.pdf

Gismero, E. (2010). EHS, Escala de Habilidades Sociales. TEA EdicionesU.

Hernández, R. (2014). Metodología de la Investigación. Sexta Edición

Moscoso, J. (2010). Guía de sesiones. Habilidades para la vida. Para organizaciones juveniles.

Patón, R., Ferreira, B., \& García, J. (2018). Incidencia de los juegos cooperativos en el autoconcepto físico de escolares de educación primaria. Retos: nuevas tendencias en educación física, deporte y recreación., (34), 14-18.

Ruiz, V., \& Jaramillo, E. (2010). Habilidades sociales en estudiantes de psicología de la Universidad Cooperativa de Colombia, sede Montería. Revista Pensando Psicología, 6(11), 53-63.

Sailema, M., López, P., Pérez, M., Cosquillo, J., Sailema, A., \& Vaca, M. (2017). El Autoconcepto y la educación física en estudiantes de secundaria. Revista Cubana de Investigaciones Biomédicas, 36(3), 31.

Tacca, D., Cuarez, R., \& Quispe, R. (2020). Habilidades Sociales, Autoconcepto y Autoestima en Adolescentes Peruanos de Educación Secundaria. International Journal of Sociology of Education, 9(3), 293-324. Obtenido de https://hipatiapress.com/hpjournals/index.php/rise/article/view/5186

Zumba, D. (2017). Disfuncionalidad familiar como factor determinante de las habilidades sociales en adolescentes de la Fundación Proyecto Don Bosco. Bachelor`s thesis, Pontificua Universidad Católica del Ecuador.

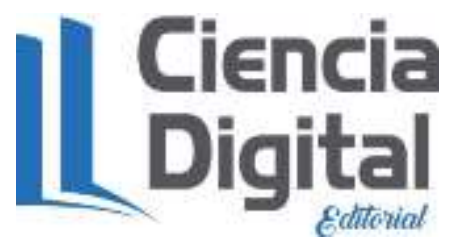




\section{PARA CITAR EL ARTÍCULO INDEXADO.}

Joseline Andrea, B. O., Indacochea Mendoza, L. R., Cadena Peralta, G. A., \& Ochoa Guallpa, M. P. (2021). El autoconcepto y las habilidades sociales de los estudiantes de Psicopedagogía (Universidad Técnica de Ambato) durante la emergencia sanitaria. Explorador Digital, 5(4), 44-64. https://doi.org/10.33262/exploradordigital.v5i4.1880

\section{Liencia}

El artículo que se publica es de exclusiva responsabilidad de los autores y no necesariamente reflejan el pensamiento de la Revista Explorador Digital.

El artículo queda en propiedad de la revista y, por tanto, su publicación parcial y/o total en otro medio tiene que ser autorizado por el director de la Revista Explorador Digital.
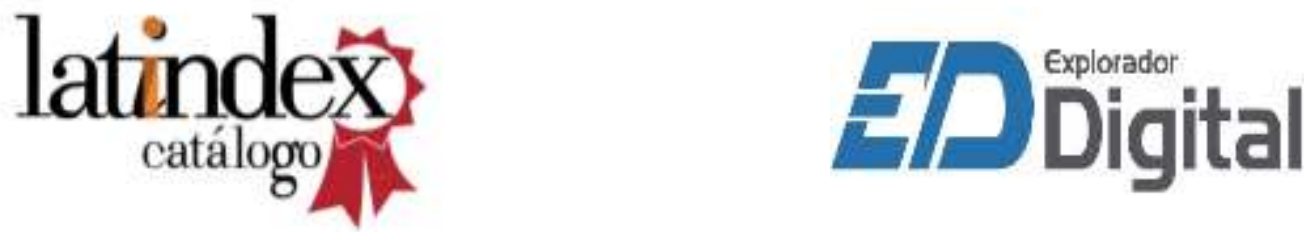